\title{
Decision Support System for the Negotiation of Bilateral Contracts in Electricity Markets
}

\author{
Francisco Silva ${ }^{1}$, Brígida Teixeira ${ }^{1}$, Tiago Pinto ${ }^{12}$, Isabel Praça ${ }^{1}$, Goreti \\ Marreiros $^{1}$, and Zita Vale ${ }^{1}$ \\ 1 GECAD - Knowledge Engineering and Decision Support Research Center, Institute \\ of Engineering - Politechnic of Porto (ISEP/IPP), Porto, Portugal * \\ 2 BISITE - Research Centre, University of Salamanca, Salamanca, Spain \\ $\{$ f spsa, bccta, tmcfp, icp, mgt, zav\}@isep.ipp.pt \\ ${ }^{2}$ tpinto@usal.es

\begin{abstract}
The use of Decision Support Systems (DSS) in he a Electricity Markets (EM) is essential to provide strate ac supp to its players. EM are constantly changing, dynamic envir n w vith many entities which give them a particularly complex nat e. Th are several simulators for this purpose, including Bilater on acting. However, a gap is noticeable in the pre-negotiatio ph se of nergy transactions, particularly in gathering information on op osin no sotiators. This paper presents an overview of existing too- for dec sion support to the Bilateral Contracting in EM, and proposes a new to l hat addresses the identified gap, using concepts related to automated negotiation, game theory and data mining.

Keywords: Automated Cegotia nilateral Contracts, Data Mining, Decision Support System. Electricity Markets, Game Theory
\end{abstract}

\section{Introduction}

Since the begi ing of the $21^{\text {st }}$ century, the EM have undergone a profound restructuring, ro ling to its liberalization. Therefore, EM became more competitive, $\mathrm{b}$ als more complex, resulting in increased unpredictability[1].

vaday - ew challenges arise regarding the increasing usage of energy from rene ar e surces. The European Union (EU) has defined a set of policies and stand rds that contribute to the large-scale implementation of distributed generation, in order to encourage and increase the use of this type of energy. An example would be the "20-20-20" program [2]. However, the usage of this energy type introduces a new source of unpredictability in the sector, due to its intermittent nature. As such, the unpredictability and risk in the EM are increasingly

\footnotetext{
* This work has received funding from the European Union's Horizon 2020 research and innovation programme under the Marie Sklodowska-Curie grant agreement No 641794 (project DREAM-GO) and grant agreement No 703689 (project ADAPT); and from FEDER Funds through COMPETE program and from National Funds through FCT under the project UID/EEA/00760/2013
} 
higher, considering the great complexity of the interaction between its participating entities and the large number of associated variables, which hinder the decision-making process. In this context, EM simulation proves to be a great tool for decision support, through the study of the inherent mechanisms of these markets as well as the relations between the entities (players), by analysing their profiles, behaviours and strategies [3]. Although there are several EM simulators, few are the ones that provide support for negotiation among players.

This paper intends to address the identified gap, aiming to propose a solution that allows participating agents to obtain the best possible results, considering their objectives. As such, it's considered a Decision Support System (DSS) for bilateral contracts, including methodologies for the pre-negotiation ph particularly in the profile analysis of opposing negotiators. This way, it all vs aa ptability of the negotiation strategies, which together with a conter alysis, gives the capability to analyse and identify different contex of hegot tion.

This paper is divided into five sections: Bilateral Contra ts alectricity Markets, Multi-Agent Simulators as Decision Support ystems in Electricity Markets, Proposal, Experimental Findings and Concly io ?

\section{Bilateral Contracts in Electri ity 1 a kets}

EM are constantly evolving and adaptins Cur ently, most countries have their own market or participate in regional markets, wgether with neighbouring countries [4]. EM are composed of severa ${ }^{1}$ narket types [5, 6], based on three different models, which are (I) day-ahe ss ot intra-day, both usually auction based, and (III) bilateral contracts models.

In the scope of EM, bila ral contracts are long-term contracts established between two entities, by no seller, for energy transaction, without the involvement of a third ent ty. T e transaction is usually carried out several weeks

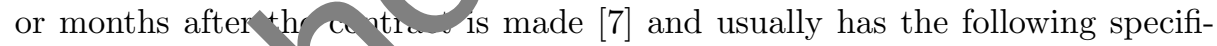
cations: start nond no and times; Price per hour (€/MWh) and amount of energy (MW, var ble throughout the contract and, finally, a range of hours relative to tho del ry of the contract. Players can use customized long-term contracts, radis of "over the counter" and electronic trading to conduct bilatera thact is [8]. There are four types of bilateral contracts: the first type are or aru Contracts, that consist in energy exchange between a buyer and a selle for a future date, for the price negotiated at that moment; the second type are Future Contracts, which are similar to Forward Contracts except that they are managed by a third party responsible for ensuring compliance with the agreement; the third type are Option Contracts, that are similar to the Forward and Future contracts with the difference that the two entities only guarantee a buy/sell option; the last one are Contracts for Difference, that allows entities concerned to protect themselves from the energy price change between the date of establishment of the agreement and the agreed date of exchange.

With the exception of Contracts for Difference, this type of negotiation allows players to control the price at which they will transact energy, in contrast to 
what happens in spot markets, due to the proposals' instability. In establishing a Forward or Future contract, players are committing themselves to transact energy for a given price at a future time, with the risk of making a transaction at a lower price than the expected and lose competitive power. Option Contracts or Contracts for Difference can avoid this risk. The first allows the player to choose not to go through with the exchange while the second ensures that the transaction is carried out at the market price. However, the first option also has the risk of not guaranteeing whether or not the other party will exercise their option to exchange and the second option does not allow better prices than the market. This way, it is possible to understand the risk associated with the negotiation of bilateral contracts and the need that players have of to that help them reduce this risk and even optimize their profits.

\section{Multi-Agent Simulators as Decision Suppo t Gotems in Electricity Markets}

The vast majority of EM simulators are focused oplw on narket analysis and are void of mechanisms that allow their users to get su pert for direct negotiation. Particularly with bilateral contracts, there ar gap in decision support systems, namely in obtaining information abou op osing party [9]. This leads to an urgent need to acquire new methods that sup the decision process, such as the definition of suitable models, choice of the best candidates to close the deal, analysis of the previous transactior of these candidates and strategies to employ to get the best deal possible. One or the main advantages of using MAS for the implementation of these sim ators is that they have software agents that, by definition, interact with o $e^{-1}$ er autonomously in order to meet an objective [10].

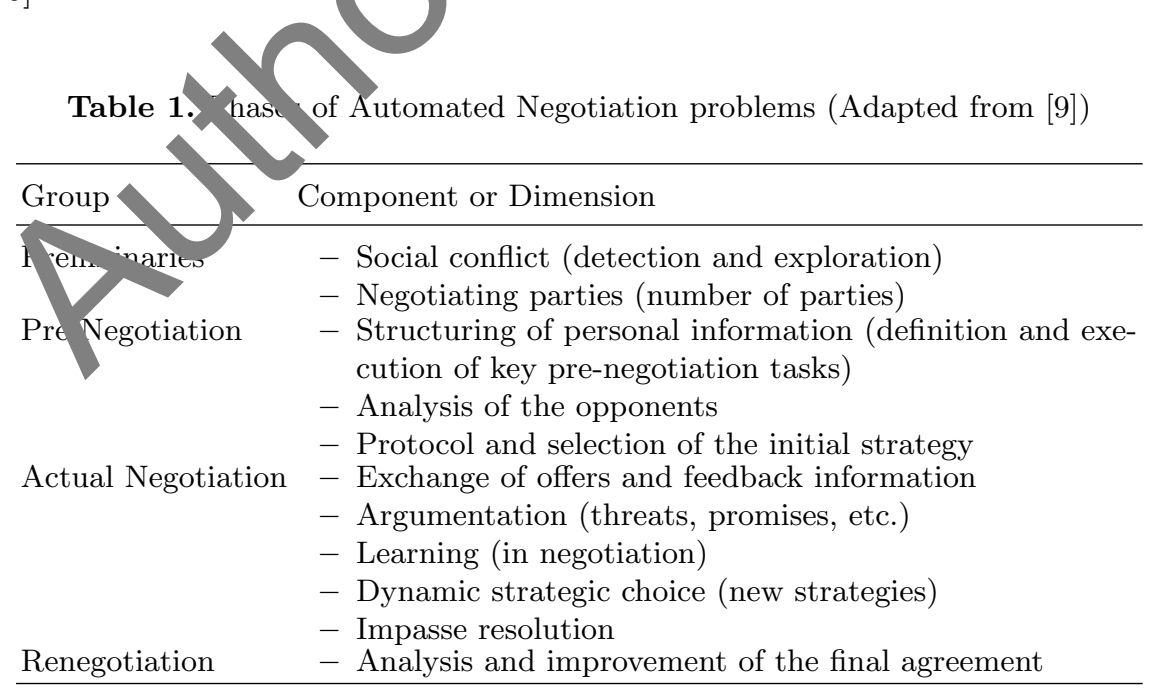


In the literature, it is possible to find references to systems that allow the simulation of bilateral contracts in the context of EM. Some examples are The Electricity Market Complex Adaptive System (EMCAS) [11], The Multi-Agent Negotiation and Risk Management in Electricity Markets (MAN-REM) [9] and The General Environment for Negotiation with Intelligent Multi-purpose Usage Simulation (GENIUS) [12]. These simulators are based on phased negotiation approaches, following the ideology of automated negotiation (see Table 1), namely in the definition of the pre-negotiation, actual negotiation and analysis of the results in order to adjust future proposals. However, these simulators do not respond to the problem already identified, that is, in the pre-negotiation phase, a detailed study of the characteristics of possible opponents does not ur, in order to perceive which are best suited to their objectives and whic neg iation strategies should be used with each one of them, in order to be best possible deal.

\section{Proposal}

This DSS focused on the Pre-Negotiation phase Jo nin the Preliminary and Pre-Negotiation phases of automated negoti tion nis union simplifies the system given the proximity of the two phases, eing ned in most models. The DSS is intended to support an agent who inten ls to transact energy with other agents through bilateral contracts. The agent supported by this system may be any type of entity interested in tran actions within the electricity markets. The agent will use the system wh nev $r$ tends to enter new bilateral contracts and wish to guarantee the ma imum $n$ ssible gain. When an agent pretends to enter into a bilateral contra market, it can find more than one competitor. Weighted competitor ch to is ry important when maximizing profits. Moreover, this decision does ot ne essarily fall on the competitor who can offer the best proposals. The co np - or's ability to comply with the agreement is a very important fac or . he fu. lment of agreements, however promising they may be, with competitur wh fail to do their part ends up being a great waste of time for the agent $\mathrm{d}$ onpromising his management.

For dec sion apport in this stage of negotiation, the system uses a scenario ana yn methu based on game theory. The outputs of this method are the choice of th $r$ ost ravourable competitors, the distribution of the amount of energy, to be ne otiated with each of the selected competitors, which guarantees greater profit, and the price that each competitor is expected to show.

As can be seen in Fig.1, the proposed method is composed of three parts: Scenarios Definition, Possible Actions Definition and Decision Process. The Scenarios Definition consists in the specification of the different negotiation scenarios that the supported agent can find. The results of the potential competitors in the past are analysed and, by means of forecast algorithms, the prices of the competitors, for different amounts of energy, are forecast. When there is not enough history to make forecasts, an estimation process is necessary. The Possible Actions Definition are the generation of all alternative actions that the supported 


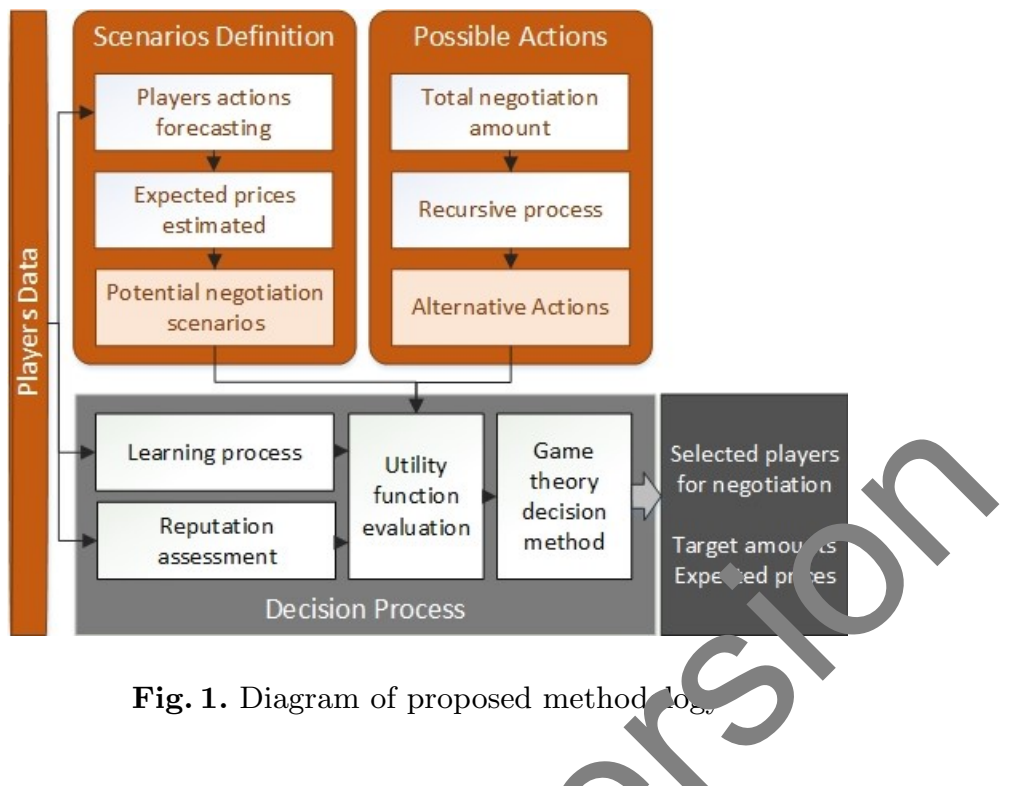

agent can take. The total energy to be nege iat with each competitor is determined through a recursive process to expl it an ossibilities. The last part, Decision Process, is composed by the crine o competitors to negotiate with, the respective amounts of energy and the expocted prices. For this purpose, a game theory approach is used to eva ate the potential outcome of each scenarioaction combination through a til to a ction. The appraisal also considers the reputation of each competitor and aloo ases a learning process, which allows to know which scenarios are mô likely to occur in each context.

Concerning the user' $\mathrm{Im}$ ra tion with the system, a graphical interface is available, allowing the ser $t$, enter his objectives and his possible competitors, obtaining a d a doution (the quantities to be negotiated with certain competitors ald th expe ted prices) coupled with an explanation of the results.

\section{Exp rim ntal Findings}

This se cion presents a case study that allows the evaluation of the proposed tool's peration. In order to facilitate the analysis of results, it's considered a simple scenario in which the supported player intends to buy $10 \mathrm{MW}$ in a weekday context. In order to obtain decision support on the opponents to negotiate, and corresponding quantities, the supported player indicates the 5 players that it can negotiate with. From his point of view, the calculation of each opponent's reputation should have as much importance to his personal opinion as the social opinion. The social opinion itself will be calculated giving the same weight to the supported group's members' opinion, as well as the group of each target player. The decision method to be used is the Most Probable, which selects the action with the highest utility of the most probable scenario, determined by the 
learning module. Finally, regarding the risk value, several tests will be carried out in order to perceive its impact.

In the first stage, a forecast is performed to obtain the price that each opponent may propose, considering their contracts history. In case of unsufficient data to forecast the price for a given quantity, a value is estimated, considering the price forecasts for the other quantities. Fig. 2 shows the forecast results.

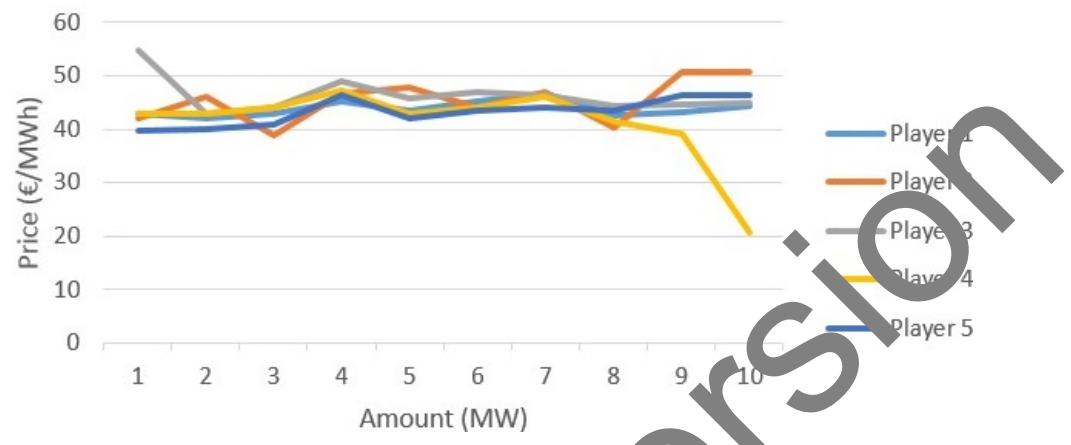

Fig. 2. Opponents expected pri per y amount

Supported by the data in the application's aatabase, it was possible to forecast the expected prices for all qua ities for all opponents, with the exception of the $10 \mathrm{MW}$ quantity. The es im ti n ade for this quantity shows prices close to those presented in the reme ning am unts of energy, except for the estimated price for Player 4. In this cas there is a deviation from his normal behaviour due to forecasts of quar 1to e. seeding $10 \mathrm{MW}$, which presented a significant downward trend. Takin into account the results of the forecast phase, Player 4 presents the 3 s st ing price, precisely for the amount of energy that the supported pla ${ }^{\circ}$ in ends o acquire. However, the final decision will also depend on each opponen's ro utation (see Table 2).

Table 2. Reputation of the opponents
\begin{tabular}{llllllll} 
Player ID & 1 & 2 & 3 & 4 & 5 \\
\hline Reputation & 0.275 & 0.575 & 0.125 & 0.325 & 0.425 \\
\hline
\end{tabular}

As it can be seen in Table 2, the player with the highest reputation is Player 2, followed by Player 5 and then Player 4 (player with the best selling price). Depending on the player's propensity to risk, reputation may not favour Player 4 against Players 2 and 5 who, despite not having such attractive sales prices, are more secure due to their reputation. To determine the best choice the sup- 
ported player can take, all possible actions are generated and the utility value of each one is calculated. The range of possible actions are the maximum number of different power distributions by the various opponents, from trading $10 \mathrm{MW}$ with only one player, to trading the same amount, but divided by several players (Example: $6 \mathrm{MW}$ with one player and $1 \mathrm{MW}$ with each of the remaining). The actions' utility value combines the reputation of the players to trade in that action, with the economic advantage it can bring to the supported player. The two components' weight varies depending on the supported player's risk propensity. The risk value ranges from 0 to 1 where 0 is the minimum risk and only the reputation component is considered and 1 is the maximum risk, where only the economic component is considered. The number of possible distrib ins of $10 \mathrm{MW}$ per 5 players is 1001, a very high number, considering the oun of energy and possible opponents in question. Through the Most $\mathrm{Pro}^{-1} \mathrm{le}$ ecision method, it was possible to obtain the negotiation recommen latic os pre ented in Fig. 3, for different risk levels.

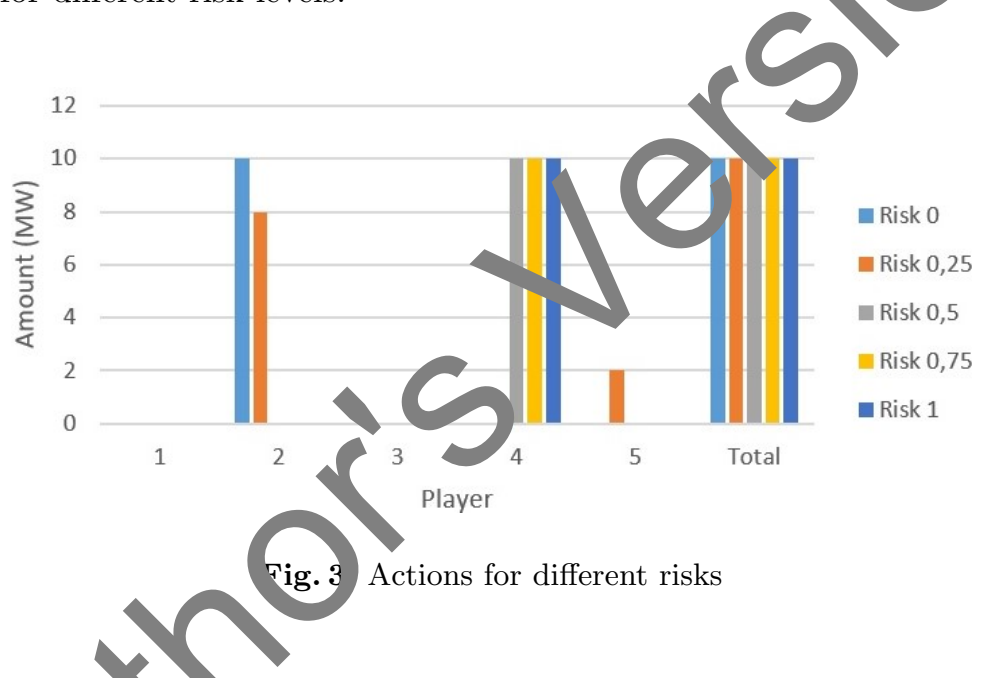

Figure 3 sho s th t in case of risk 0 , that is, each action is evaluated only by the reputa on the players involved, it's recommended to transact all the energy wiu $\mathrm{Pla}$ or 2 , the player with the highest reputation. By increasing the risk to 25, $=$ no longer advisable to negotiate all power with Player 2. The supp $\mathrm{r}^{+}$d prayer should only transact 8MW with Player 2 and $2 \mathrm{MW}$ with Player 5 (sec nd highest reputation). In this case, some security is abandoned in favour of a more economically advantageous transaction. This is followed by a case where the risk value is 0.5 , where the reputational component is as important as the economic component. In this case, since Player 4 has a superior advantage in the economic component than Players 2 and 5 in the reputation component, he's selected to transact the $10 \mathrm{MW}$. Being Player 4 in advantage, the risk increase, favouring the economic component, will keep the recommendation in his favour.

Through this case study it is possible to perceive the advantages that a player, intending to make bilateral contracts, may have when using this decision support system. The system presents the expected results according to the supported 
player's expectations and presents the best solution in the middle of a very high number of possible actions in a large number of scenarios that would otherwise be practically impossible to achieve.

\section{Conclusions}

The paper presents a state of art for bilateral contracting in EM as well as acknowledgement of automated negotiation as the main strategy for modelling this type of simulators. It was concluded that the simulators seek to follow the automated negotiation phases, but present weaknesses in not exploring the information of the opposing traders in the pre-negotiation. Thus, a DSS that $\rho$ poses a solution to the identified problem is presented, which uses data ming inniques and game theory to select the players with whom they inter nt otiate, fulfilling their requirements. A study was performed in order to c mon, trate the advantages of the tool for the decision maker.

As future work, alternative approaches to the Possibl Action phase will be considered, to present an higher performance while kee ng ne pality of results.

\section{References}

1. M. Shahidehpour, H. Yamin, Z. Li, and John Viley $\propto$ Sons., Market operations in electric power systems : forecasting, schew in $q$, and risk management. Institute of Electrical and Electronics Engineers, Wiley-Interscience, 2002.

2. European Commission, "The 2020 -imate and energy package," 2009.

3. H. Li and L. Tesfatsion, "De relo me f Open Source Software for Power Market Research: The AMES Test Bed," Jour al of Energy Markets, 2009.

4. F. P. Sioshansi, Evolution Global electricity markets : new paradigms, new challenges, new approaches. Flse ier Science \& Technology, 2013.

5. F. Silva, B. Teixeira Г. I nto, G. Santos, Z. Vale, and I. Praça, "Generation of realistic scenarios m m ti-agent simulation of electricity markets," Energy, vol. 116, pp. o\& 13 . dec 2016.

6. G. B. Sheb aputawonal Auction Mechanisms for Restructured Power Industry Operation 1 ton, MA: Springer US, 1999.

7. H. Algarv an Lopes, Risk Management and Bilateral Contracts in Multiagent \ectr ty Markets, pp. 297-308. Cham: Springer International Publishing,

8. . S r r schen and G. Strbac, Fundamentals of power system economics. John Ley \& Sons, 2004.

9. F. opes, T. Rodrigues, and J. Sousa, "Negotiating Bilateral Contracts in a Multiagent Electricity Market: A Case Study," in 2012 23rd International Workshop on Database and Expert Systems Applications, pp. 326-330, 2012.

10. M. Luck and M. D"Inverno, "Agency and Autonomy: A Formal Framework," tech. rep., Coventry, UK, UK, 1995.

11. V. Koritarov, "Real-world market representation with agents," IEEE Power and Energy Magazine, vol. 2, pp. 39-46, jul 2004.

12. R. Lin, S. Kraus, T. Baarslag, D. Tykhonov, K. Hindriks, and C. M. Jonker, "GENIUS: An Integrated Environment for Supporting the Design of Generic Automated Negotiators," Computational Intelligence, vol. 30, pp. 48-70, feb 2014. 